\title{
MODIFICATIONS ON RF COMPONENTS IN THE LCLS INJECTOR*
}

\author{
C.Limborg-Deprey, L. Xiao, D. H. Dowell, Z.Li, J.Schmerge \\ MS 18, SLAC 2575 Sand Hill Road, Menlo Park CA, U.S.A.
}

\section{Abstract}

Design of the first generation LCLS injector is nearing completion. Fabrication has begun and component installation is planned for 2006. We discuss the last modifications made on both the 1.6 cell S-Band RF gun and the SLAC S-Band accelerating structures to minimize irreversible emittance growth. The mode separation between the 0 and $\pi$ modes was increased from $3.4 \mathrm{MHz}$ to $15 \mathrm{MHz}$. Dual feed and racetrack shapes have been incorporated in the full cell of the new gun. The linac sections were also modified to accommodate dual feeds and racetrack shapes in their input cells. PARMELA simulations indicating the need for these modifications are presented.

\section{INTRODUCTION}

The commissioning of the LCLS PhotoInjector beamline will start in January 2007. Final design modifications on RF components were incorporated in 2005 prior to fabrication. Many of the modifications were based on operational experience with the prototype 1.6 cell RF S-Band gun [1]. Additional geometry modifications were made at the recommendation of SLAC rf experts [2] to reduce peak fields at the rf coupler. The radius of the first iris was made larger to increase the mode separation between the 0 and $\pi$ modes from $3.4 \mathrm{MHz}$ to $15 \mathrm{MHz}$. The rf coupling slot was modified from theta to Z-coupling. The full cell tuners were modified to push-pull deformable wall tuners to eliminate the penetration in the cavity wall. A dual rf feed and racetrack shape in the full cell have been included. Cooling channels have been added to handle a $4 \mathrm{~kW}$ average power [3] although with proper rf pulse shaping the average power should not exceed $1.8 \mathrm{~kW}$ [4]. At the end of this paper, we also briefly explain the need to implement dual feeds in the input cells of the linac structure.

\section{RF GUN}

\section{Increased Mode Separation}

The RF gun is a two cell device and therefore has two resonant modes. These modes are characterized by their 0 and $\pi$ phase shifts between the two cells. The $\pi$ mode (accelerating mode) is resonant at $2856 \mathrm{MHz}$ and the 0mode is resonant at $2852.5 \mathrm{MHz}$ in the prototype gun. The $\pi$ mode is approximately $550 \mathrm{kHz}$ wide with a loaded $\mathrm{Q}$ of over 5000 and the 0 mode is $400 \mathrm{kHz}$ wide with a loaded Q of nearly 7000 . When the gun is excited at 2856 $\mathrm{MHz}$, the field is dominated by the $\pi$ mode however the 0 -mode is also excited due to finite resonant width of the

\footnotetext{
* Work supported by the U.S. DOE Contract No. DE-AC02-76SF00515
} Contact: limborg@slac.stanford.edu
0 -mode and finite bandwidth of the driving pulse. The amplitude of the 0 -mode in the half cell is less than $10 \%$ of the $\pi$ mode amplitude in the prototype gun. By increasing the mode separation to $15 \mathrm{MHz}$, this value is reduced to less than $3 \%$. Since the 0 mode is nearly $90^{\circ}$ out of phase with the $\pi$ mode, the additional field does not decrease the rf emittance term. The main effect of the 0 mode is to alter the phase shift between cells from $180^{\circ}$ when there is no 0 mode to approximately $176^{\circ}$ degrees in the prototype gun. Simulations show that the presence of the 0 mode increases slightly the emittance.

\section{Beam dynamics in the presence of $0-\pi$ mode}

Simulations were done using PARMELA. The nominal parameters of $1 \mathrm{nC}, 10 \mathrm{ps}$ long and $1.2 \mathrm{~mm}$ radius laser pulse and $0.72 \mathrm{~mm} . \mathrm{mrad}$ cathode emittance were used. Emittance was optimized principally scanning solenoid and injection phase, i.e. laser timing with respect to the phase of the accelerating field ( $\pi$-mode). Different maps of field were studied with mode separation ranging from 3.4 MHz to $20 \mathrm{MHz}$. Figure 1 compares simulation results of the prototype gun $(3.4 \mathrm{MHz})$ and the LCLS gun $(15 \mathrm{MHz})$. Shown is the total projected emittance as a function of the phase difference between the 0-mode and the $\pi$-mode in the $1 / 2$ cell. The optimal "baseline" emittance was computed assuming a 0 voltage field in the 0 -mode. A $0.94 \mathrm{~mm} . \mathrm{mrad}$ emittance was obtained for the $15 \mathrm{MHz}$ and $0.99 \mathrm{~mm} . \mathrm{mrad}$ for the $3.4 \mathrm{MHz}$. For a 90 degrees relative phase difference and the nominal field voltages of $12 \mathrm{MV} / \mathrm{m}$ and $3 \mathrm{MV} / \mathrm{m}$, the best emittances obtained were respectively of $0.975 \mathrm{~mm} . \mathrm{mrad}$ for the prototype gun and $0.945 \mathrm{~mm}$.mrad for the LCLS gun.

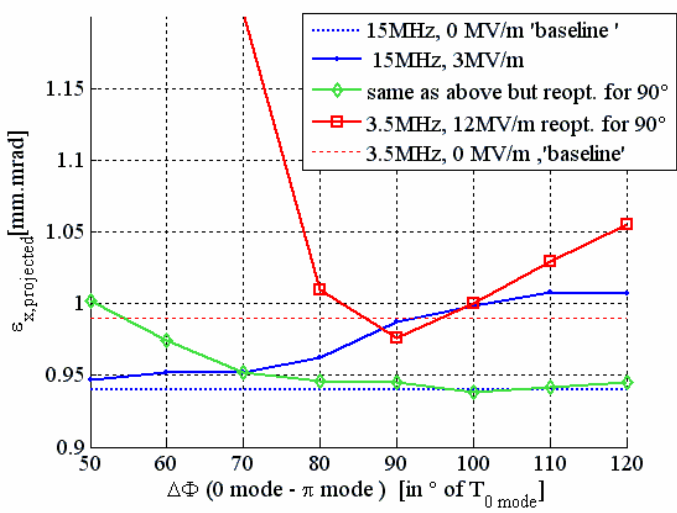

Figure 1: Projected emittance vs phase difference between the $\pi$ and 0 -modes. The baseline levels correspond to the optimum emittance assuming no 0 -mode field.

The beamline was then reoptimized for the cases including the 0 -mode at its nominal voltage and for a 90 degrees phase difference. The emittance degradation as a function of the relative phase difference is much larger for 
the $3.4 \mathrm{MHz}$ mode separation case than for the $15 \mathrm{MHz}$ one. The range of phase difference explored, between 40 and 130 degrees, is justified in the next paragraph.

By adopting the $15 \mathrm{MHz}$ mode separation, we suppress another degree of freedom in an already very large parameter space. We also reduce dramatically the RF emittance from $0.28 \mathrm{~mm} . \mathrm{mrad}$ to $0.15 \mathrm{~mm} . \mathrm{mrad}$. This improvement could be very beneficial in a regime in which the RF emittance contribution would represent a large fraction of the total emittance.

A systematic RF emittance determination, using ellipsoidal pulses, as described in [5], should have been performed. However, it was more relevant at this stage to study the gun performances using the laser pulse shape available at the early stage of the LCLS operation. The $\mathrm{RF}$ emittance numbers were deduced assuming that the different emittance contributions sum in quadrature.

\section{Dynamics of the 0 - $\pi$ modes}

Both the $\pi$ and 0 -modes are excited by the RF input due to finite resonance width of the modes and finite bandwidth of the klystron pulse. The $\pi$ - and 0 -modes couple to the external drive respectively with $\beta=2$ for the $\pi$-mode and $\beta=0.88$ for the 0 -mode. The excitation of the modes in the cavity has been simulated using the SLED cavity model [6]. Figure 2 shows the time domain excitation of the $\pi$ and 0 -modes with two different RF pulses: $3-\mu$ s square pulse and $0.82-\mu$ s shaped pulse.
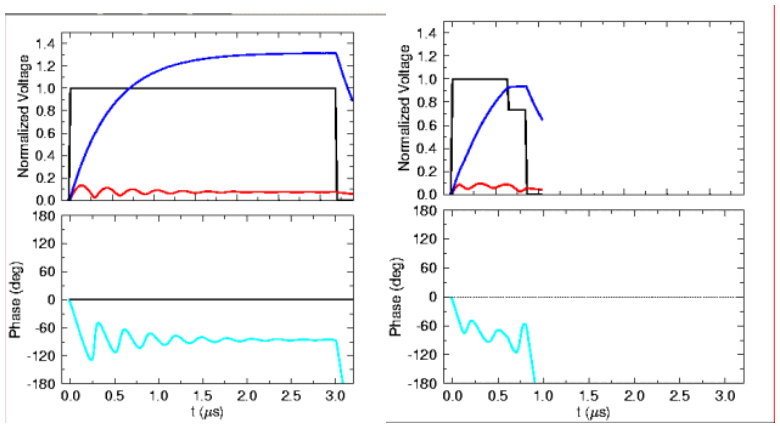

Figure 2: Amplitude and Phase Difference of the $\pi$ and 0modes developing with time for two different klystron pulse shapes (in black) ; (a) for a square $3 \mu$ s long pulse ; (b) for a $0.8 \mu \mathrm{s}$ klystron pulse with power stepping down from $30 \mathrm{MW}$ to $13 \mathrm{MW}$ after $0.6 \mu \mathrm{s}$ was assumed

Table 1: Cathode field for the 0-mode for different mode separation studied at steady state

\begin{tabular}{|l|l|}
\hline \multicolumn{1}{|c|}{0 - $\pi$ mode $(\mathrm{MHz})$} & 0 -mode cathode field \\
\hline 3.4 & $10 \%$ \\
\hline 8.0 & $5 \%$ \\
\hline 15.0 & $3 \%$ \\
\hline 20.0 & $2 \%$ \\
\hline
\end{tabular}

Using the emitted field information and the RF parameters of the modes obtained using the field solver, the cathode field of the 0 -mode can be calculated. The ratio of the 0 and $\pi$ mode cathode fields for four different designs is shown in the Table 1.

Once steady state is reached, and when driven with a long klystron pulse, the 0 -mode phase is close to 90 degrees ahead of that of the $\pi$ mode in the half cell. When driven with the short and shaped pulse, the 0-mode is still in transition when the $\pi$ mode reaches the designed voltage. The phase difference between the 0 and $\pi$ modes can vary between 40 and 130 degrees once the field level is reached.

\section{Geometry changes}

The $15-\mathrm{MHz}$ mode separation is achieved by modifying the disk of the prototype Gun to a thinner disk with a larger iris opening. This allowed the increase in the radius of curvature and consequently reduces the surface field at the iris. In the new gun, the surface field never exceeds the cathode field. In the prototype gun, the surface field was up to $10 \%$ larger than the cathode field at the iris.

The increased mode separation also makes the system less sensitive to changes in the field balance with the gun's body temperature.

\section{Dual Feed}

The prototype gun has a single rf feed in the full cell of the gun[7]. An identically sized and shaped hole is machined 180 degrees opposite the coupler hole to reduce the dipole field in the prototype gun. However the presence of the coupler hole and the symmetrizing port induces quadrupole and higher order fields in the coupler cell due to a four-fold symmetry geometry. In addition, the single rf feed can only compensate for the amplitude term, and the full cell field will still have phase induced multipoles due to the gradient in phase introduced by $\mathrm{rf}$ power flowing from only one side of the cell, which are not compensated in the prototype design [8].

The dual feed eliminates the time-dependent dipole field but the quadrupole component remains at a similar level assuming identical coupling holes as used in the single feed gun. A racetrack cell geometry was then incorporated in the new design to minimize the quadrupole field [9].

A dual feed with proper racetrack geometry eliminates both the dipole and quadrupole fields. The higher order modes are small and negligible.

\section{Racetrack Shape}

The coupling apertures are designed with coupling coefficient $\beta$ of 2 , the quadrupole moment $\gamma \beta_{\perp}$ introduced by the couplers is about $4.4 \mathrm{e}-3 / \mathrm{mm}$. In order to correct the quadrupole components, a racetrack cavity shape has been designed. The center offsets of the two arcs are adjusted to minimize the quadrupole field. In the present LCLS gun design, this is reduced to less than $8 \mathrm{e}-5 / \mathrm{mm}$.

The skew quadrupole moment introduced by the laser ports in the half cell was calculated to be approximately $3.8 \mathrm{e}-4 / \mathrm{mm}$. The reduced quadrupole fields in both full and half cells in the new design does not cause significant degradation to the emittance as shown below. 
PARMELA simulations show that without a racetrack shape in the full cell, the beam would acquire a quadrupole head-tail moment. This quadupole moment would lead to an irreversible emittance growth of $0-20 \%$ from the nominal $0.9 \mathrm{~mm}$.mrad slice emittance at the head of the bunch as shown in figure 3. The ratio of horizontal to vertical beam sizes would also vary along the bunch, making the beam matching more difficult in the linac. These simulations show that the racetrack shape entirely suppresses the head-tail quadrupole moment for our nominal beam parameters.

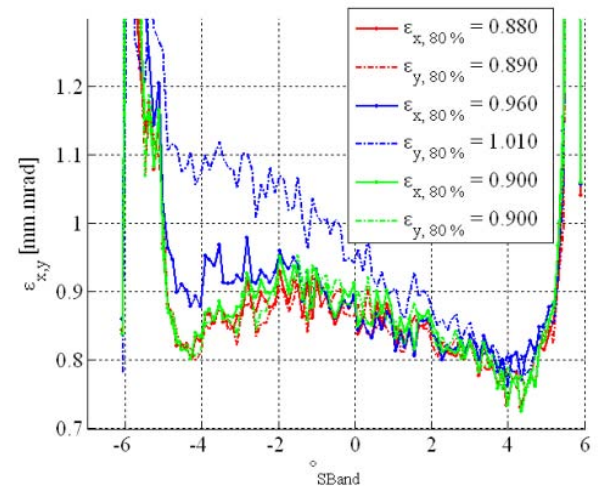

Figure 3: Hor./Ver. slice emittance for 100 slices; the $80 \%$ emittance, i.e., the projected emittance for the 80 central slices is given in the legend- the three cases shown are (1) red - 2D geometry (cylindrical symmetry)- (2) blue$3 \mathrm{D}$ geometry -with dual feed but no racetrack shape - (3)green- 3D geometry - includes all ports and racetrack shape

\section{LINAC STRUCTURES}

\section{Dual feed}

In the S-Band SLAC accelerating structures, the single feed input and output couplers produce transverse time dependent forces. We were essentially concerned with the dipole and quadrupole moments of those forces. The effect of higher order moments is negligible.

The constant gradient structure imposes variable diskloaded irises and cell radii. The head-tail kick is thus much smaller when the beam goes through the output cell.

The emittance growth depends on the betatron function at the kick location following equation (1).

$$
\frac{\Delta \varepsilon}{\varepsilon_{o}}=\sqrt{1+\frac{\sigma_{(\gamma \beta)_{\perp}}^{2} \beta_{\text {twiss }}}{\gamma \varepsilon_{n}}}-1
$$

The correction of those head-tail kicks by steering the beam and using the transverse wakefield could have reduced the problem but not solved it, in particular, for our low charge $0.2 \mathrm{nC}$ operation.

The geometry changes, including the modification of the input cell length to match the WR384 waveguide port length, are described in [10].
Table 2: Emittance growth induced by head-tail kicks- (a) LOa Entrance- (b) L0a Exit - (c) L0b Entrance - (d) LOb Exit

\begin{tabular}{|c|l|l|l|l|}
\hline$(\Delta \varepsilon / \varepsilon)$ at $0^{\circ}$ in $\%$ & (a) & (b) & (c) & $(\mathrm{d})$ \\
\hline single feed & $\mathbf{1 . 8}$ & 0.4 & $\mathbf{1 2}$ & $0.6 / 0.5$ \\
\hline dual feed & $\mathbf{5 . 1 0}$ & 0.4 & $\mathbf{0 . 0 4}$ & $0.6 / 0.5$ \\
\hline
\end{tabular}

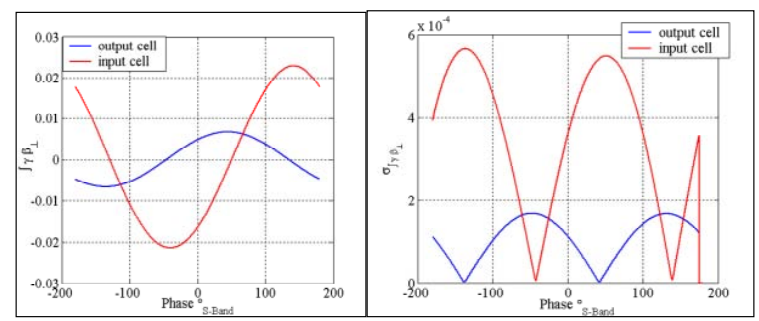

Figure 4: For input and output coupler cells on a 10ps long pulse (a) Transverse Dipole kick as a function of Linac phase for a $20 \mathrm{MV} / \mathrm{m}$ accelerating field- (b)- rms head tail kick -

\section{Acknowledgements}

We would like to thank T.Smith for suggesting the mode separation study and J.Rose for valuable discussion.

\section{REFERENCES}

[1] D.Palmer PhD thesis "The next Generation Photoinjector", June 1998, Stanford University

[2] J.Wang, Report of the LCLS Injector Technical Review Committee, SLAC Memo, dated November 22, 2004, http://www-ssrl.slac.stanford.edu/lcls/ photoinjector/internals/internals.html\#RF

[3] R.F. Boyce et al. "Design Considerations for the LCLS RF Gun.", LCLS-TN-04-4

[4] J.Schmerge et al., "Reducing the Heat Load on the LCLS $120 \mathrm{~Hz}$ RF Gun with RF Pulse Shaping", LCLS-TN-02-7

[5] C.Limborg-Deprey et al. "Alternate Tunings for the Linac Coherent Light Souce", these proceedings

[6] Z.D.Farkas, "SLED: A Method of doubling SLAC's Energy", SLAC-PUB-1453

[7] D.T.Palmer, et al ," Cold Test Results of the SLAC/BNL/UCLA 1.6 Cell Photocathode RF Gun", SLAC-PUB-6799

[8] G.Loew et al., " The Stanford 2-mile accelerator ", P147, R.Neal Editor, Stanford University , 1968

[9] L.Xiao et al. "Dual Feed RF Gun Design for the LCLS" these proceedings

[10]Z.Li et al. "Coupler Design for the LCLS Injector SBand Structure " these proceedings 